\title{
The ICP22 protein selectively modifies the transcription of different kinetic classes of pseudorabies virus genes
}

\author{
Irma F Takács, Dóra Tombácz, Beáta Berta, István Prazsák, Nándor Póka and Zsolt Boldogkői*
}

\begin{abstract}
Background: Pseudorabies virus (PRV), an alpha-herpesvirus of swine, is a widely used model organism in investigations of the molecular pathomechanisms of the herpesviruses. This work is the continuation of our earlier studies, in which we investigated the effect of the abrogation of gene function on the viral transcriptome by knocking out PRV genes playing roles in the coordination of global gene expression of the virus. In this study, we deleted the us 1 gene encoding the ICP22, an important viral regulatory protein, and analyzed the changes in the expression of other PRV genes.
\end{abstract}

Results: A multi-timepoint real-time RT-PCR technique was applied to evaluate the impact of deletion of the PRV us 1 gene on the overall transcription kinetics of viral genes. The mutation proved to exert a differential effect on the distinct kinetic classes of PRV genes at the various stages of lytic infection. In the us 1 gene-deleted virus, all the kinetic classes of the genes were significantly down-regulated in the first hour of infection. After 2 to $6 \mathrm{~h}$ of infection, the late genes were severely suppressed, whereas the early genes were unaffected. In the late stage of infection, the early genes were selectively up-regulated. In the mutant virus, the transcription of the iel80 gene, the major coordinator of PRV gene expression, correlated closely with the transcription of other viral genes, a situation which was not found in the wild-type (wt) virus. A 4-h delay was observed in the commencement of DNA replication in the mutant virus as compared with the wt virus. The rate of transcription from a gene normalized to the relative copy number of the viral genome was observed to decline drastically following the initiation of DNA replication in both the wt and mutant backgrounds. Finally, the switch between the expressions of the early and late genes was demonstrated not to be controlled by DNA replication, as is widely believed, since the switch preceded the DNA replication.

Conclusions: Our results show a strong dependence of PRV gene expression on the presence of functional us 1 gene. ICP22 is shown to exert a differential effect on the distinct kinetic classes of PRV genes and to disrupt the close correlation between the transcription kinetics of ie180 and other PRV transcripts. Furthermore, DNA replication exerts a severe constraint on the viral transcription.

Keywords: Herpesvirus, Pseudorabies virus, Real-time PCR, ICP22, us 1 gene

\footnotetext{
*Correspondence: boldogkoi.zsolt@med.u-szeged.hu

Department of Medical Biology, Faculty of Medicine, University of Szeged, Somogyi B. st. 4, Szeged H-6720, Hungary
} 


\section{Background}

The pseudorabies virus (PRV), an alpha-herpesvirus, is the etiological cause of Aujeszky's disease of swine [1]. PRV is related to the human pathogen varicella-zoster virus (VZV) and herpes simplex virus types 1 and 2 (HSV-1 and -2), and the animal herpesvirus bovine herpesvirus type 1 (BHV-1). PRV is widely used as a model organism in investigations of the molecular pathomechanisms of the herpesviruses [2], and is a useful tool for the mapping of neural circuits [3,4]. Attempts have additionally been made to utilize this virus as a gene delivery vector [5,6] and an oncolytic agent [7]. Besides the lytic phase, alpha-herpesviruses can enter a latent state, where they transcribe a limited set of cis-antisense RNAs [8]. Traditionally, the lytic herpesvirus genes are classified into three kinetic categories: immediate-early (IE) genes, early (E) genes and late (L) genes. On a finer scale, an intermediate category, the early/late $(E / L=$ delayed early) genes can also be distinguished [9]. PRV encodes a single IE gene, the ie180 gene. The IE180 protein, a transactivator, is the principal coordinator of the overall gene expression of the virus. E genes encode proteins required for the nucleotide metabolism and DNA replication. Other E genes such, as the early protein $0(e p 0)$ [10] and $u l 54$ genes [11], encode transcriptional regulators. Most of the $\mathrm{L}$ genes code for structural elements of the virus. ICP22 is one of the five IE proteins of HSV-1, which is encoded by the us1 gene. Intriguingly, a large part of the HSV us1 gene is located in the unique US region, whereas its promoter and a short 5' portion of the transcribed region are in the inverted repeat (IR) segment. In PRV, however, the entire us1 gene (earlier called the rsp 40 gene) resides in the IR region; this gene is therefore represented in two copies in the PRV genome. There is no consensus as to whether the PRV us1 gene is expressed in IE [12] or E kinetics [13]. We demonstrated in an earlier analysis that this gene is expressed in atypical kinetics, and that it is obviously not an IE gene [9]. The function of the ICP22 polypeptide has primarily been analyzed in HSV-1. The investigations have revealed that ICP22 is a multifunctional protein that plays roles in various aspects of HSV pathogenesis. It has not yet clearly established whether ICP22 acts to repress E genes [14] or to enhance the transcription of L genes [15]. It has been shown that not all $\mathrm{L}$ genes require this transactivator for their expressions [16]. The BICP22 protein of BHV-1, a homologue of ICP22, has been demonstrated to exert a general repressive effect on each kinetic class [17]. Rice and coworkers reported that ICP22 acts at the level of transcriptional regulation [18]. However, the level of ICP0 mRNA was also reduced in the us1 knockout (KO) HSV [16], which raises the question of whether the direct cause of the reduced transcription is the lack of us1 gene activity or the low ICP0 mRNA level. ICP22 has to be phosphorylated by the viral UL13 protein kinase in order to accomplish the transcriptional activation of $\mathrm{L}$ genes [19]. An additional function of the ICP22 polypeptide is associated with the alteration of the activity of cyclin-dependent kinase cdc2, a regulator of the cell cycle, which results in a selective up-regulation of HSV L genes during lytic infection [20]. Furthermore, HSV ICP22 also acts to modify the phosphorylation of RNA polymerase II (RNAP II) [21], which carries out the transcription of viral genes. One of the major control regions of RNAP II is its carboxy terminal domain (CTD), residing on the large subunit of the molecule. The CTD, containing multiple repeats of a heptapeptide sequence, serves as a binding site for various cellular proteins involved in the regulation of transcription. ICP22 is presumed to trigger the loss of Ser-2 phosphorylation on the CTD, and thereby modify the activity of RNAP II [22]. A novel function of ICP22 was recently identified, involving alteration of the chaperon localization of the host cells [23]. It has been shown that ORF63, the ICP22 homolog of VZV, does not alter RNAP II phosphorylation and the host chaperon machinery [22], which might indicate that ICP22 acts in a species- or genus-specific manner. In the present study, we have investigated the effects of us1 gene deletion on the overall transcription of PRV genes.

\section{Results and discussion}

\section{Experimental design}

An insertion mutant PRV strain was constructed which contains the mutation in both copies of the us1 gene.

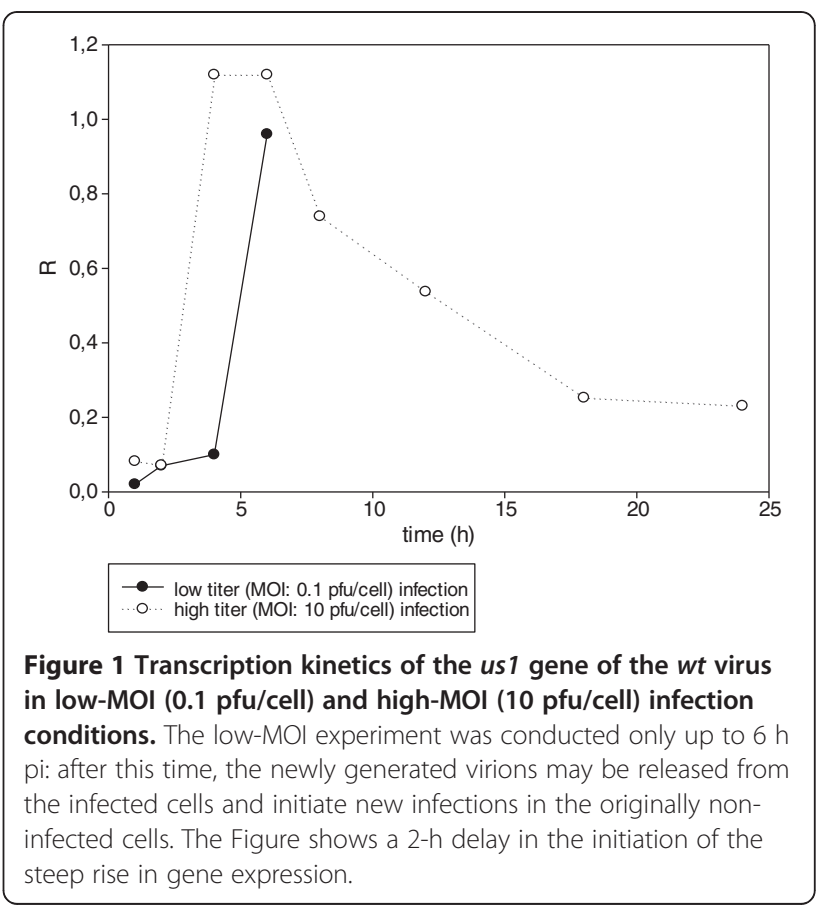


Mutation of a DNA sequence in the internal repeat region is copied to the terminal repeat by a mechanism called equalization [24]. From among the 70 PRV genes, 32 were selected for the transcription analysis, which reside at the upstream position of the tandem gene clusters and which represent each kinetic class of PRV genes. The reason for this choice was to exclude the distorting effect of the transcriptional read-through exerted by the upstream genes on the downstream genes. Furthermore, the genes selected for analysis play important roles in the regulation of the overall gene expression of the virus, including the $i e 180$, the ep0 (and their antisense transcripts, the LAT and the AST, respectively), the virion host shut-off (vhs), and the ul54 genes. For each viral gene, a minimum of 3 parallel replicates were performed in order to achieve statistical reliability. Immortalized PK-15 cells were infected with either the wild-type ( $w t)$ or us1 gene-deleted (us1-KO) PRV, using a high multiplicity of infection [MOI; 10 plaque-forming units (pfu)/cell]. The low-dose infections produce a much finer resolution of the cycle threshold
(Ct) values for the transcripts than in the case of highdose infections; however, in the former case a large proportion of the cells remain uninfected, which allows the initiation of an additional infection cycle after $6 \mathrm{~h}$ postinfection (pi) by the newly generated virions [9], which would confuse the interpretation of the expression data obtained. The transcription of PRV genes was monitored at 9 different time points: $0.5,1,2,4,6,8,12,18$ and $24 \mathrm{~h}$ pi (multi-timepoint analysis). Strand-specific primers were used for the reverse transcription reaction so as to exclude the distorting effects of the potential cisantisense transcripts that might be produced from the complementary DNA strands, which cannot be avoided on the use of other methods, such as oligo-dT- or random priming-based reverse transcription [25]. On the other hand, we found that the specificity of strandspecific primer-based RT is much higher than that of other methods [9]. In our work, we applied a modified version of the mathematical model described by Soong and colleagues for the relative quantification [26]. Specifically, we used the average of the 6-h ECt-sample values

\section{$\mathbf{R}_{\mathrm{r}}$ values}

\begin{tabular}{|c|c|c|c|c|c|c|c|c|c|c|}
\hline Genes & 0,5 & 1 & 2 & 4 & 6 & 8 & 12 & 18 & 24 & \\
\hline ie180 & 0,030 & 0,109 & 0,207 & 0,204 & 0,266 & 2,819 & 4,129 & 6,547 & 3,342 & \\
\hline epo & 0,069 & 0,218 & 1,497 & 2,118 & 4,149 & 10,077 & 12,184 & 8,912 & 3,651 & \\
\hline ul9 & 0,007 & 0,439 & 4,095 & 1,410 & 1,351 & 5,517 & 4,987 & 5,529 & 5,439 & \\
\hline ul21 & 1,589 & 0,555 & 0,547 & 0,213 & 0,245 & 0,313 & 0,738 & 1,357 & 0,881 & \\
\hline ul23 & 0,011 & 0,011 & 0,066 & 0,087 & 0,307 & 0,643 & 1,053 & 1,205 & 0,390 & \\
\hline ul29 & 0,033 & 0,052 & 0,191 & 0,266 & 0,375 & 1,653 & 2,395 & 2,561 & 1,413 & \\
\hline ul30 & 0,019 & 0,121 & 2,274 & 1,360 & 0,835 & 4,170 & 4,329 & 4,608 & 2,785 & \\
\hline ul50 & 0,015 & 0,086 & 1,716 & 0,859 & 1,152 & 3,067 & 3,402 & 3,174 & 1,240 & 1300 \\
\hline ul52 & 0,188 & 0,245 & 0,685 & 2,489 & 2,721 & 7,148 & 2,778 & 6,524 & 1,371 & \\
\hline ul54 & 0,012 & 0,018 & 0,211 & 0,154 & 0,151 & 0,490 & 0,527 & 0,765 & 0,404 & 9,000 \\
\hline us3 & 0,024 & 0,258 & 0,409 & 0,873 & 2,078 & 5,036 & 7,050 & 12,059 & 3,780 & 6,000 \\
\hline ul14 & 0,009 & 0,326 & 1,114 & 0,779 & 0,987 & 4,246 & 3,032 & 2,668 & 2,597 & 3,000 \\
\hline ul15 & 0,012 & 0,009 & 0,075 & 0,101 & 0,127 & 0,319 & 0,721 & 1,076 & 0,565 & 1,000 \\
\hline ul43 & 0,022 & 0,417 & 1,495 & 0,913 & 1,225 & 2,382 & 9,553 & 6,690 & 2,234 & 900 \\
\hline ul53 & 0,429 & 1,347 & 1,007 & 0,488 & 0,706 & 0,511 & 1,106 & 3,220 & 1,891 & 0 \\
\hline us6 & 0,005 & 0,013 & 0,080 & 0,070 & 0,113 & 0,097 & 0,129 & 0,457 & 0,419 & 0,800 \\
\hline ul1 & 0,021 & 0,108 & 0,214 & 0,263 & 0,428 & 0,504 & 1,046 & 3,082 & 0,937 & 0,700 \\
\hline ul5 & 0,015 & 0,005 & 0,003 & 0,023 & 0,132 & 0,060 & 0,324 & 0,249 & 0,322 & 0,600 \\
\hline ul6 & 0,433 & 0,308 & 0,055 & 0,077 & 0,144 & 0,166 & 1,070 & 1,254 & 0,808 & 0,500 \\
\hline ul10 & 0,043 & 0,023 & 0,071 & 0,117 & 0,244 & 0,220 & 0,358 & 0,816 & 0,444 & 0,400 \\
\hline ul17 & 0,068 & 0,025 & 0,062 & 0,066 & 0,175 & 0,298 & 0,634 & 1,178 & 0,885 & \\
\hline ul19 & 0,488 & 0,091 & 0,185 & 0,159 & 0,250 & 0,285 & 1,136 & 2,517 & 1,563 & 0,300 \\
\hline ul22 & 0,031 & 0,400 & 0,281 & 0,530 & 0,257 & 1,750 & 2,679 & 8,338 & 3,160 & 0,200 \\
\hline ul24 & 1,929 & 1,246 & 1,430 & 0,264 & 0,512 & 1,112 & 2,001 & 3,925 & 0,948 & 0,100 \\
\hline ul32 & 0,052 & 0,028 & 0,073 & 0,117 & 0,288 & 0,316 & 0,734 & 1,855 & 1,264 & 0,0 \\
\hline ul33 & 0,008 & 0,331 & 0,484 & 0,243 & 0,284 & 0,611 & 1,807 & 2,123 & 1,424 & \\
\hline ul36 & 0,052 & 0,061 & 0,645 & 0,091 & 0,150 & 0,322 & 0,632 & 1,670 & 1,031 & \\
\hline ul37 & 0,305 & 0,034 & 0,205 & 0,021 & 0,225 & 0,314 & 0,868 & 1,578 & 1,414 & \\
\hline ul41 & 0,244 & 0,258 & 0,817 & 0,584 & 0,790 & 3,305 & 1,998 & 3,334 & 1,155 & \\
\hline ul42 & 0,053 & 0,156 & 0,388 & 0,351 & 0,977 & 1,460 & 1,534 & 1,784 & 1,673 & \\
\hline ul44 & 0,015 & 0,005 & 0,007 & 0,009 & 0,021 & 0,044 & 0,082 & 0,960 & 0,380 & \\
\hline ul48 & 0,031 & 0,084 & 0,126 & 0,109 & 0,195 & 0,263 & 0,386 & 2,211 & 1,557 & \\
\hline ul51 & 0,451 & 0,008 & 0,001 & 0,099 & 0,070 & 0,419 & 0,197 & 0,264 & 0,245 & \\
\hline LAT & 0,230 & 0,095 & 0,172 & 0,074 & 0,101 & 0,159 & 0,247 & 0,558 & 0,270 & \\
\hline AST & 0,124 & 0,081 & 0,116 & 0,018 & 0,100 & 0,278 & 0,600 & 1,443 & 1,062 & \\
\hline
\end{tabular}

Figure 2 Heatmap visualization of the ratios of the transcripts of the us1-KO and the wt viruses $\left(\mathbf{R}_{\mathbf{r}}=\mathbf{R}_{\mathrm{us} 1 \mathrm{Ko}} / \mathbf{R}_{\mathrm{wt}}\right)$. The red color $\left(\mathrm{R}_{\mathrm{r}}<1\right)$ indicates a transcript level that is lower in the mutant than in the wt virus at a certain period of infection, whereas the black $\left(R_{r}>1\right)$ indicates the opposite: the mutant virus produces a higher amount of mRNA than the wt virus. 


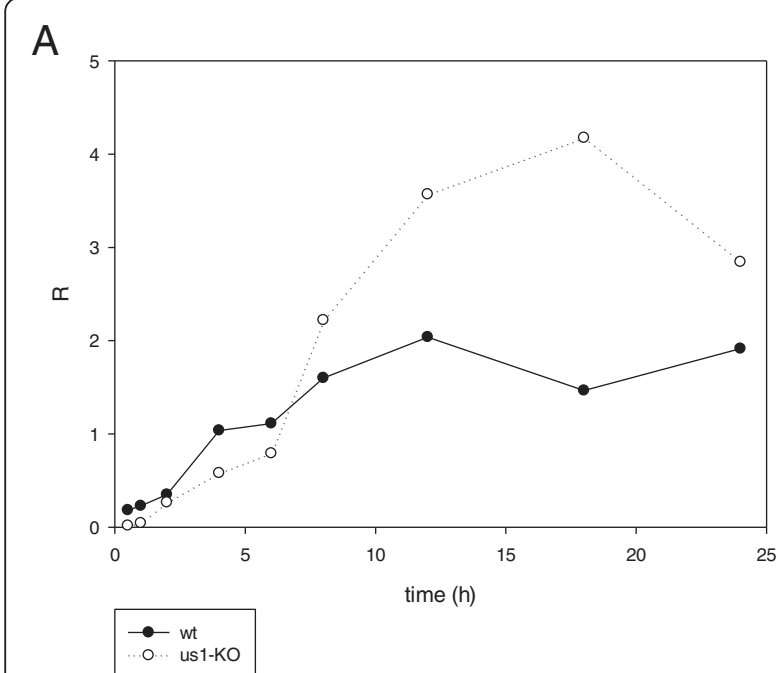

B

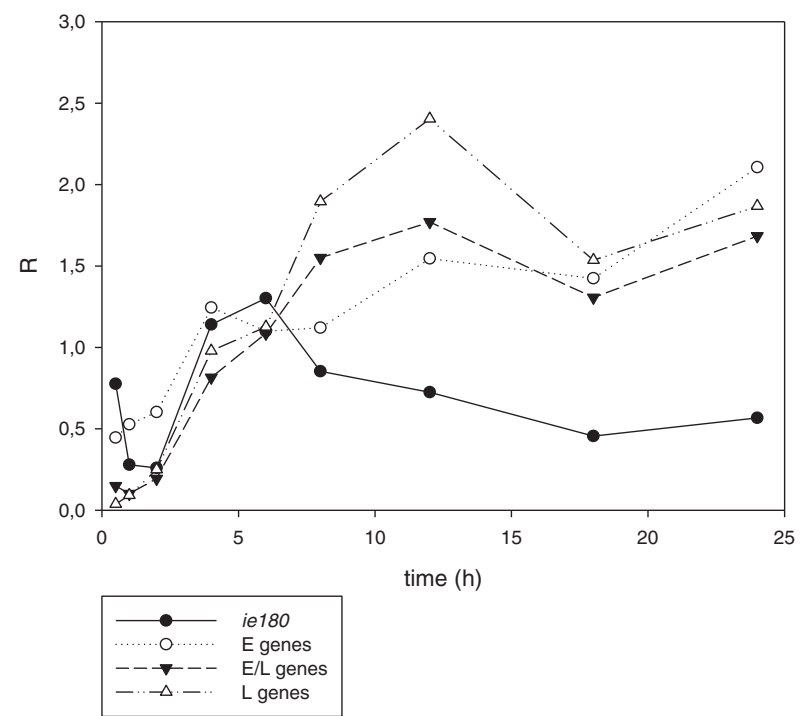

us1-KO

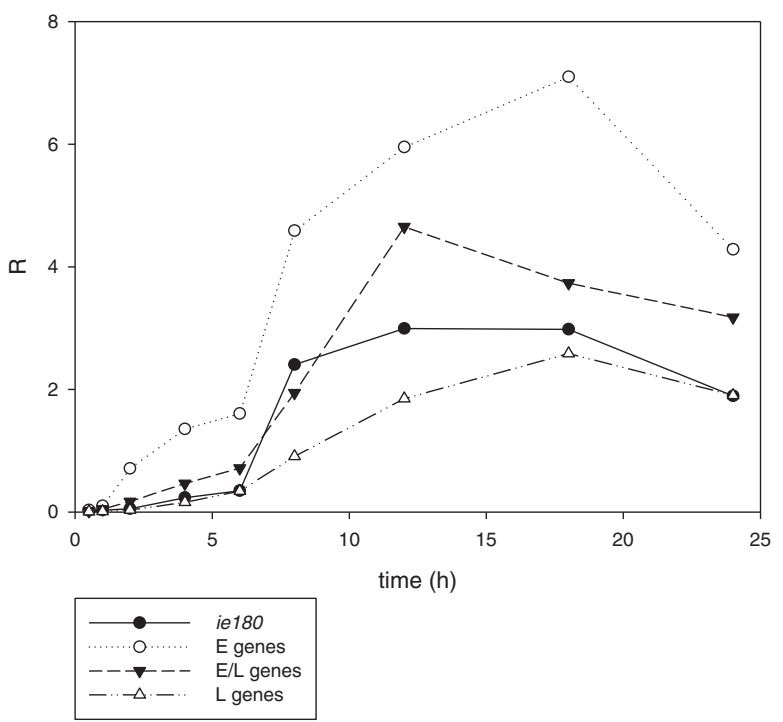

Figure 3 The average relative expression ratios $(\overline{\boldsymbol{R}})$. (A) The $\bar{R}$ values of the total genes of the mutant and wt viruses plotted against time. The average level of the transcripts of the mutant virus is lower in the early stage, but higher in the late stage of infection. (B) The $\bar{R}$ values of the different kinetic classes of mutant and wt viruses. In the mutant virus, the $L$ genes are repressed in the early stage of infection, whereas the $E$ gene expressions are enhanced in the late stage of infection.

of the $w t$ PRV for each gene in both the $w t$ and the mutant backgrounds, as controls, which were normalized to the average of the corresponding $28 \mathrm{~S}$ values (ECtreference). The $28 \mathrm{~S}$ RNA gene was used as a reference gene since the ribosomal RNAs are not substrates of VHS ribonuclease [27]. We used a selected $\mathrm{Ct}$ value (at $6 \mathrm{~h}$ in our system) as control for the comparability of the relative copy numbers of a transcript at different time points. The relative amounts of the transcripts of different genes cannot be compared directly due to the variation in the primer efficiencies in both RT and PCR. However, the use of multi-timepoint qPCR analysis allows a comparison of the transcription kinetics of different viral genes throughout the whole period of infection. Furthermore, this method allows a comparison of the same genes in the two genetic backgrounds $\left(R_{r}=\right.$ $\left.R_{\mathrm{us} 1 \mathrm{KO}),,} R_{\mathrm{wt}}\right)$, and of the mRNAs and the complementary antisense transcripts in the case of the ep0/LAT and ie180/AST pairs. A high $\mathrm{R}_{\mathrm{r}}$ value indicates an inhibitory effect of the ICP22 protein on the transcript level of a particular gene in the $w t$ virus. Conversely, a low $\mathrm{R}_{\mathrm{r}}$ value indicates a stimulatory effect on the gene expression. We applied the same logic for the interpretation of the data obtained as is used in other knockout organisms, i.e. the normal role of the us1 gene is considered to be the opposite of that of the phenotype 


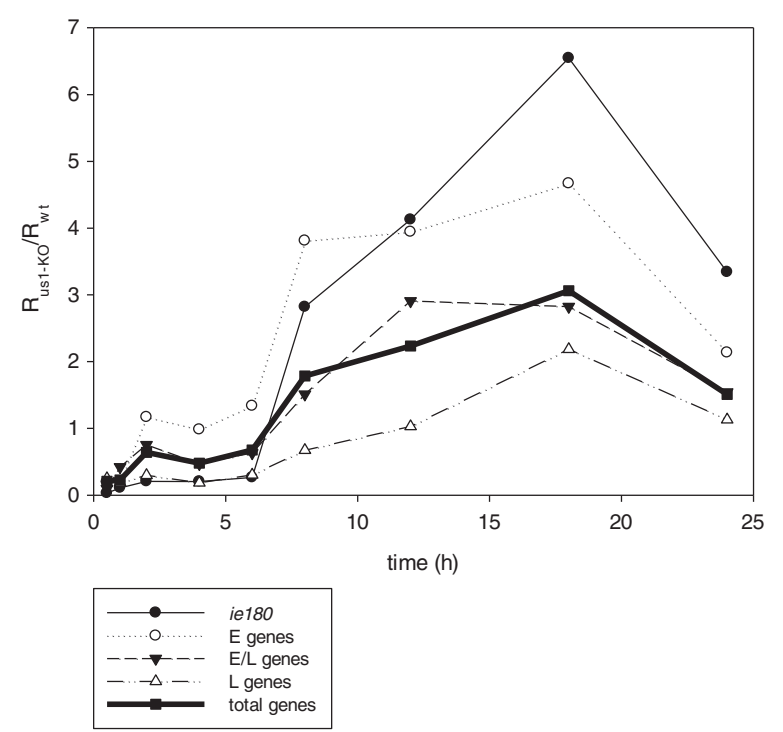

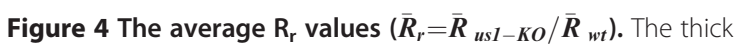
solid line depicts the $\bar{R}_{r}$ values of the total genes, and the thinner lines the $\bar{R}_{r}$ values of the different kinetic classes of viral genes.

caused by the mutation of this gene. In other words, an elevated expression of a gene in the us1-null mutant is indicative of a suppressive effect of the ICP22 protein on the expression of this gene.

\section{Confirmation of the mutant genotype}

The mutation in the us1 gene was confirmed by PCR amplification of the DNA sequences containing the mutation, followed by pyrosequencing. The mutation of the us1 gene was rescued, and this was followed by growth analysis in order to confirm that the altered kinetic properties can be solely explained by the abrogation of the us1 function (data not shown). Besides these analyses, we also carried out more precise techniques for this purpose. Thus, we compared the rates of increase of viral DNA during the first twelve hours of viral infection. We observed similar dynamics in the growth rates of the DNA of the wild-type and rescued viruses, while both differed significantly from those of the us1-mutant virus (Additional file 1). In addition, we compared the transcription kinetics of the two most important transactivator genes, the ie 180 and ep0 genes of pseudorabies virus. This revealed that the kinetics of the rescued virus resembled that of the wild-type virus, but differed significantly from that of the mutant virus (Additional file 2 and Additional file 3).

\section{Transcription of the us1 gene in the wild-type PRV}

We compared the expression kinetics of the us1 gene of the $w t$ virus under the low- and high-dose infection conditions (Figure 1). Infection was analyzed for $6 \mathrm{~h}$ in the low-MOI infection, and for $24 \mathrm{~h}$ in the high-MOI infection. The data revealed that in the low-MOI infection the us1 gene behaved as an L gene [9], since the amounts of its transcripts started to rise rapidly from $4 \mathrm{~h}$ pi. This is in contrast with the situation of the highdose experiment where this gene exhibited typical $\mathrm{E}$ characteristics in the early phase of infection, since its expression began to rise steeply from $2 \mathrm{~h}$ pi. Thus, there was a 2-h shift in the expression kinetics between the two experimental conditions up to $6 \mathrm{~h}$ pi. Another unusual feature of the us1 mRNA kinetics was the rapid drop in the transcript level after $6 \mathrm{~h} \mathrm{pi}$, which is not typical in any of the kinetic classes of the viral genes.

\section{The impact of the us 1 gene mutation on the viral transcriptome}

The $R$ values of individual genes were calculated for both $w t$ and $u s 1-K O$ mutant viruses, and compared by calculation of the ratios $\left(R_{r}=R_{u s 1 K O} / R_{w t}\right)$ for each time point (Figure 2). The average $R$ values were calculated and plotted for the total genes $(\bar{R})$ (Figure 3A; Additional file $4 \mathrm{~A})$ and for each kinetic class of genes $\left(\bar{R}_{E}, \bar{R}_{E / L}\right.$ and $\bar{R}_{L}$.) in both genetic backgrounds (Figure 3B; Additional file $4 \mathrm{~B}$ ). Additionally, the mutant and $w t$ viruses were compared by calculating the average $R_{r}$ values for the total genes $\left(\bar{R}_{r}\right)$ and separately for each kinetic class $\left(\bar{R}_{r-E}, R_{r-E / L}\right.$ and $\left.\bar{R}_{r-L}\right)$ (Figure $\left.4 \mathrm{~B}\right)$. Figures $3 \mathrm{~A}$ and 4 reveal a significant decrease in the amounts of transcripts in each kinetic class of genes in the first hour of infection (Figure 2). The average levels of transcripts were not decreased significantly or at all in a few genes (e.g. ul21, ul24, ul53 and ul19), but in most genes, and especially the E genes, the fold of the decrease was close to 2 orders of magnitude at this very early stage of infection. However, the average level of the $\mathrm{E}$ transcripts of the mutant virus reached approximately the same level as that of the $w t$ virus in the interval 1-6 h pi. This was in contrast with the L genes, which on average were still expressed at a significantly lower level during the first $8 \mathrm{~h}$ of infection. The average amounts of the E/L transcripts falls between those of the $\mathrm{E}$ and $\mathrm{L}$ genes. The ie180 gene was also expressed at a lower level in the first $6 \mathrm{~h}$ of infection in the mutant virus. The deletion of the us1 gene led to a significant reduction in the average expression of the $\mathrm{L}$ genes and to a lesser reduction in the expression of the E/L genes in the first $6 \mathrm{~h} \mathrm{pi}$, but the $\mathrm{E}$ genes were expressed at a slightly higher level in the mutant than in the $w t$ background in the period $2-6 \mathrm{~h}$ pi (Additional file 4B). The average rate of transcription of the IE, $\mathrm{E}$ and $\mathrm{E} / \mathrm{L}$ genes from the mutant viral genome exceeded the rate of transcription from the $w t$ genome after $6 \mathrm{~h}$ pi (Figure 4). This was in contrast with the transcript levels of the average $\mathrm{L}$ genes of the mutant virus, which remained below or equal to that of the $w t$ 


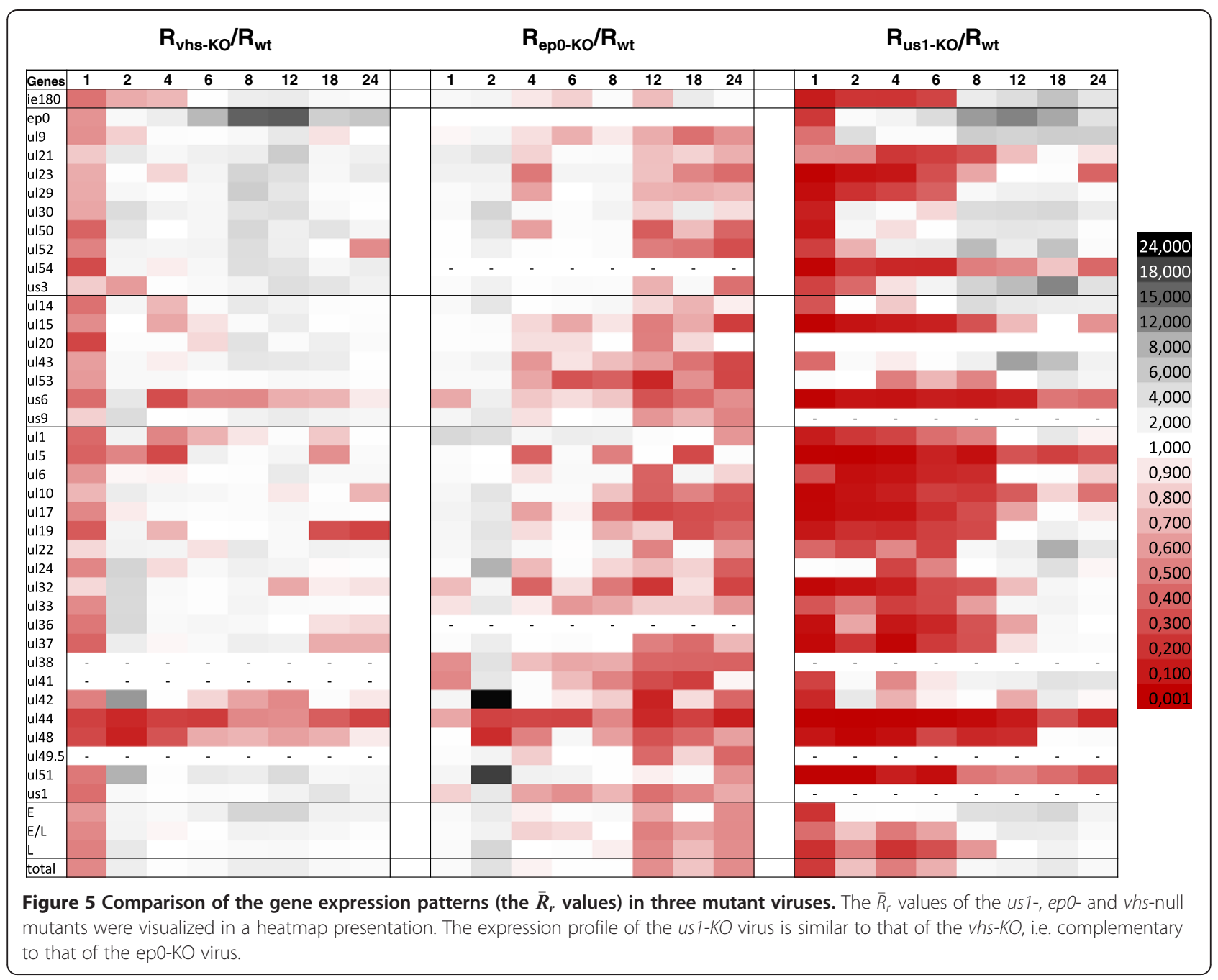

virus, except at $18 \mathrm{~h}$ pi. These data suggest that the ICP22 protein exerts an inhibitory effect especially on the transcription of the ie180 and E genes following the initiation of viral DNA synthesis. The us1 gene deletion exerted the highest impact on the expression of the ep0 gene, an important regulator of PRV gene expression [28]: the amount of ep0 transcripts was $\sim 10$ times higher in the cells infected with the mutant than that for the $w t$ virus within the period $8-18 \mathrm{~h}$ pi. This result was the opposite of that described in the HSV, where the level of ICP0 mRNA was significantly lower in the KO virus [16]. The expressions of the us3, ul22, ul43, and ul52 genes were also significantly elevated at certain time points in the late stage of infection (Figure 2). The $u l 5, u l 51$ and LAT genes were the only examined PRV genes whose expressions were always lower in the us1$K O$ virus than in the $w t$ virus. Overall, the above data suggest that the ICP22 protein exerts a selective effect on the expressions of PRV genes belonging in different kinetic classes. ICP22 (possibly of tegument origin) appeared to have a significant stimulatory effect on the general gene expression of the virus at both $30 \mathrm{~min}$ and $1 \mathrm{~h}$ pi [PRV ICP22 has been shown to be localized in the viral tegument layer [29]. Though to a lower extent, its stimulatory effect continued up to $6 \mathrm{~h}$ in the $\mathrm{E} / \mathrm{L}$ and $\mathrm{L}$ genes, but the effect became neutral or slightly inhibitory in the later stages of infection in these kinetic classes of genes. The expressions of the $E$ genes exhibited different profiles on average in the mutant background: no discernible effect of the mutation within the $1-6 \mathrm{~h}$ pi period, followed by a profound inhibitory effect in the later phases of infection. Our results may resolve the debate as to whether ICP22 represses the E genes [14] or enhances the L genes [15]. The data demonstrate that the rates of transcription of the $\mathrm{L}$ genes in the mutant virus are repressed (and therefore they are selectively enhanced in the $w t$ virus) in the very early phase of infection, whereas the rates of transcription of 


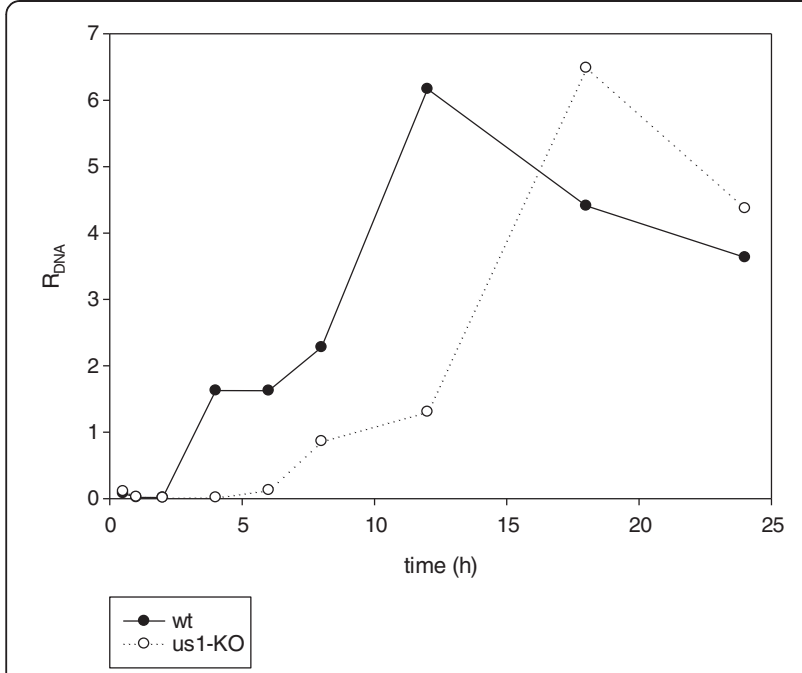

Figure 6 Synthesis of viral DNAs. The replication of viral DNA was monitored in the wt and the us 1-KO viruses through the use of realtime RT-PCR. A 4-h delay was observed in the onset of DNA replication. the E genes are selectively enhanced (and therefore they are repressed in the $w t$ virus) in the late phase of infection.

\section{Comparison of the effects on gene expression in three mutant PRV strains}

We compared the gene expression profiles ( $\bar{R}_{r}$ values) of three mutant viruses: the vhs-KO [30], epO-KO [28] and $u s 1-K O$ viruses (Figure 5). On a broad scale, the us1 and $v h s$ gene deletions appeared to produce a similar overall expression pattern (though the us1 deletion exerted a more marked effect), whereas ep 0 deletion led to an overall expression profile complementary to those of the us1$K O$ and epO-KO viruses. Figure 5 reveals that the us 1 and $v h s$ gene deletions resulted in down-regulation of the PRV genes in the $\mathrm{E}$ phase and in up-regulation of these genes in the L phase, whereas a reverse expression pattern was observed in the ep 0 mutant background. Furthermore, in both the us1-KO and the vhs-KO viruses, the most affected gene was the ep 0 , which was significantly upregulated from $2 \mathrm{~h}$ pi. These results suggest a fundamental

\section{$R_{\mathrm{rn}}$ values}

\begin{tabular}{|c|c|c|c|c|c|c|c|c|c|c|}
\hline Genes & 0,5 & 1 & 2 & 4 & 6 & 8 & 12 & 18 & 24 & \\
\hline ie180 & 0,022 & 0,076 & 0,254 & 19,072 & 3,377 & 7,423 & 19,501 & 4,451 & 2,778 & \\
\hline ep0 & 0,050 & 0,152 & 1,837 & 197,640 & 52,775 & 26,534 & 57,543 & 6,059 & 3,034 & \\
\hline ul9 & 0,005 & 0,305 & 5,025 & 131,548 & 17,190 & 14,526 & 23,555 & 3,759 & 4,520 & \\
\hline ul21 & 1,142 & 0,385 & 0,672 & 19,865 & 3,111 & 0,823 & 3,485 & 0,923 & 0,732 & \\
\hline ul23 & 0,008 & 0,007 & 0,082 & 8,128 & 3,900 & 1,694 & 4,975 & 0,819 & 0,324 & \\
\hline ul29 & 0,024 & 0,036 & 0,235 & 24,844 & 4,772 & 4,353 & 11,313 & 1,741 & 1,174 & \\
\hline ul30 & 0,014 & 0,084 & 2,791 & 126,895 & 10,615 & 10,980 & 20,447 & 3,133 & 2,315 & \\
\hline ul50 & 0,011 & 0,059 & 2,106 & 80,191 & 14,653 & 8,077 & 16,067 & 2,158 & 1,030 & \\
\hline ul52 & 0,135 & 0,170 & 0,841 & 232,261 & 34,617 & 18,823 & 13,118 & 4,436 & 1,140 & 240,000 \\
\hline ul54 & 0,009 & 0,013 & 0,259 & 14,409 & 1,918 & 1,290 & 2,490 & 0,520 & 0,336 & 120,000 \\
\hline us3 & 0,018 & 0,179 & 0,502 & 81,454 & 26,433 & 13,261 & 33,298 & 8,199 & 3,142 & 60,000 \\
\hline ul14 & 0,007 & 0,226 & 1,368 & 72,674 & 12,557 & 11,181 & 14,321 & 1,814 & 2,158 & 30,000 \\
\hline ul15 & 0,009 & 0,006 & 0,092 & 9,417 & 1,614 & 0,840 & 3,406 & 0,732 & 0,469 & 15,000 \\
\hline ul43 & 0,016 & 0,290 & 1,835 & 85,166 & 15,581 & 6,272 & 45,115 & 4,549 & 1,856 & 13,000 \\
\hline ul53 & 0,308 & 0,935 & 1,235 & 45,486 & 8,986 & 1,347 & 5,222 & 2,189 & 1,572 & 7,500 \\
\hline us6 & 0,004 & 0,009 & 0,098 & 6,574 & 1,438 & 0,255 & 0,608 & 0,311 & 0,348 & 3,750 \\
\hline$\overline{\text { ul1 }}$ & 0,015 & 0,075 & 0,262 & 24,556 & 5,444 & 1,328 & 4,941 & 2,096 & 0,779 & 1,875 \\
\hline ul5 & 0,011 & 0,004 & 0,003 & 2,133 & 1,676 & 0,159 & 1,530 & 0,170 & 0,267 & 1,000 \\
\hline ul6 & 0,311 & 0,214 & 0,06 & 7,188 & 1,825 & 0,438 & 5,056 & 0,853 & 0,671 & 0,750 \\
\hline ul10 & 0,031 & 0,016 & 0,087 & 10,959 & 3,100 & 0,579 & 1,689 & 0,555 & 0,369 & 0500 \\
\hline ul17 & 0,049 & 0,018 & 0,077 & 6,181 & 2,227 & 0,785 & 2,995 & 0,801 & 0,736 & 0,500 \\
\hline ul19 & 0,351 & 0,063 & 0,227 & 14,821 & 3,182 & 0,750 & 5,366 & 1,711 & 1,299 & 0,400 \\
\hline ul22 & 0,022 & 0,278 & 0,345 & 49,404 & 3,272 & 4,609 & 12,653 & 5,669 & 2,626 & 0,300 \\
\hline ul24 & 1,386 & 0,865 & 1,755 & 24,638 & 6,506 & 2,928 & 9,451 & 2,669 & 0,788 & 0,200 \\
\hline ul32 & 0,037 & 0,020 & 0,090 & 10,961 & 3,662 & 0,832 & 3,465 & 1,261 & 1,051 & \\
\hline ul33 & 0,005 & 0,230 & 0,593 & 22,626 & 3,614 & 1,609 & 8,532 & 1,444 & 1,183 & \\
\hline ul36 & 0,037 & 0,042 & 0,791 & 8,484 & 1,904 & 0,848 & 2,983 & 1,135 & 0,857 & \\
\hline ul37 & 0,219 & 0,024 & 0,251 & 1,981 & 2,857 & 0,828 & 4,101 & 1,073 & 1,175 & \\
\hline ul41 & 0,175 & 0,179 & 1,003 & 54,503 & 10,046 & 8,702 & 9,438 & 2,267 & 0,960 & \\
\hline ul42 & 0,038 & 0,109 & 0,476 & 32,725 & 12,425 & 3,844 & 7,245 & 1,213 & 1,391 & \\
\hline $\mathrm{ul} 44$ & 0,011 & 0,004 & 0,009 & 0,876 & 0,272 & 0,116 & 0,390 & 0,653 & 0,316 & \\
\hline $\mathrm{ul} 48$ & 0,022 & 0,058 & 0,154 & 10,206 & 2,480 & 0,692 & 1,824 & 1,503 & 1,294 & \\
\hline$\underline{u l} 51$ & 0,324 & 0,006 & 0,002 & 9,196 & 0,889 & 1,104 & 0,933 & 0,180 & 0,204 & \\
\hline$\overline{\text { LAT }}$ & 0,165 & 0,066 & 0,211 & 6,924 & 1,281 & 0,419 & 1,165 & 0,379 & 0,225 & \\
\hline AST & 0,089 & 0,057 & 0,142 & 1,638 & 1,274 & 0,732 & 2,832 & 0,981 & 0,883 & \\
\hline
\end{tabular}

Figure 7 Heatmap visualization of the ratios of the transcripts of the us 1-KO and the wt viruses normalized to the relative copy number of the DNAs $\left(\mathbf{R}_{\mathbf{r n}}=\mathbf{R}_{\mathbf{u s} 1 \mathrm{Ko}}: \mathbf{R}_{\mathrm{DNA} \text {-us } 1 \mathrm{Ko}} / \mathbf{R}_{\mathbf{w t}}: \mathbf{R}_{\mathrm{DNA}-\mathbf{w t}}\right)$. The $\mathrm{R}_{\mathrm{rn}}$ values were very low in the first $2 \mathrm{~h}$ pi. There was a drastic change in the period 4-6 $\mathrm{h}$ pi, when the $\mathrm{R}_{\mathrm{rn}}$ values became high. In the last stage of infection, the gene expressions became the same in the two viruses. 


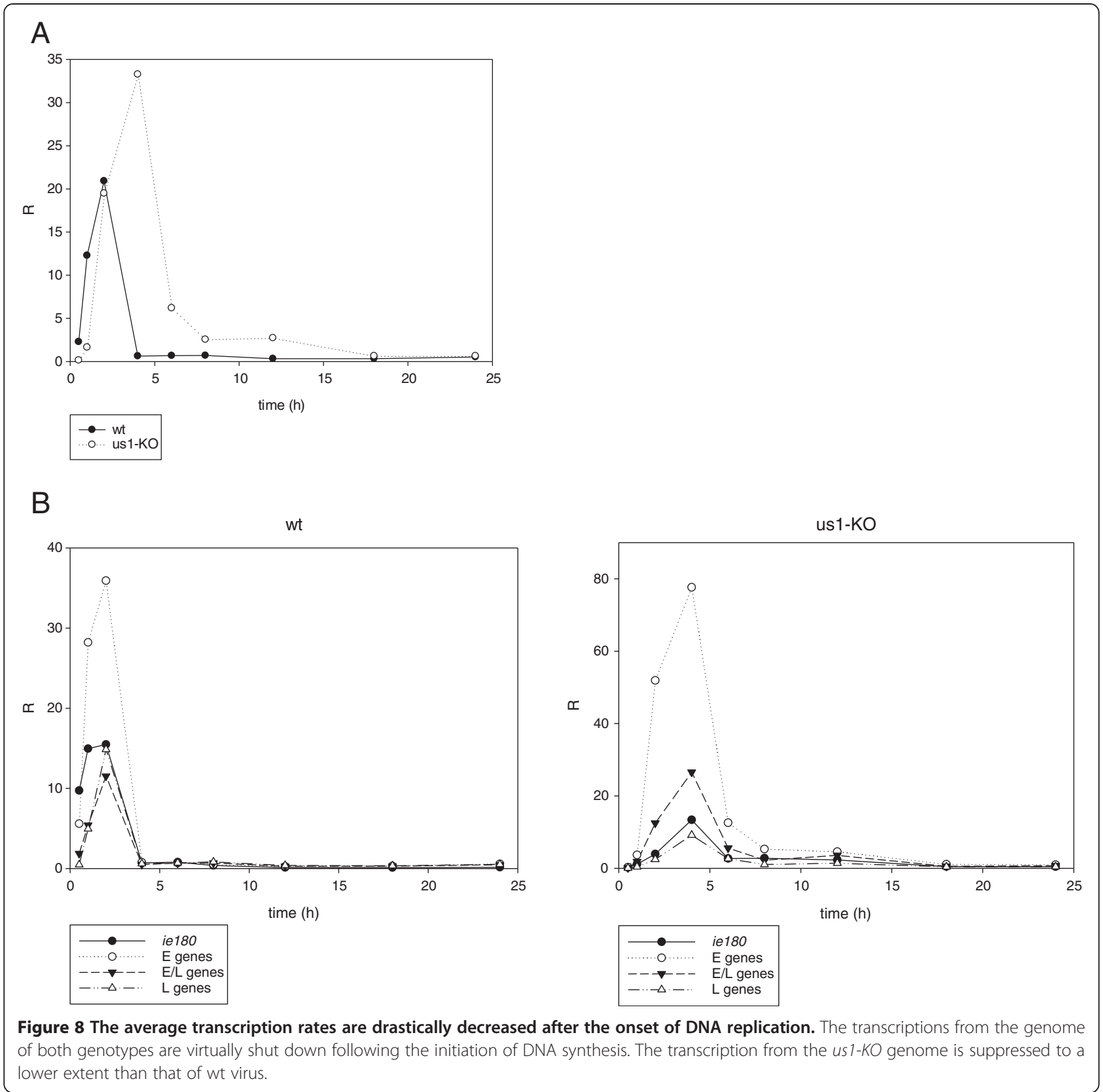

role of the EP0 transactivator in the formation of the global gene expression profile of PRV.

The expression of the ie180 gene is correlated with the expressions of the PRV genes in the us1-KO virus

We previously reported that the expression of the ie180 gene, the major transactivator of the $w t$ virus, was uncorrelated with the expressions of the remaining genes [30]. When we investigated this relationship in the us1-KO virus by using Pearson correlation analysis, very high correlations emerged between the ie180 transcripts and the transcripts produced by all three kinetic classes of the PRV genes (Additional file 5A). The correlation was especially high between the ie180 and the E transcripts. Interestingly, we observed a similar effect in the vhs-null mutant as concerns of the correlation between the ie180 and the other viral genes [30].

The initiation of DNA replication is delayed in the us 1-KO as compared with the wt virus

Our real-time RT-PCR investigation of the kinetics of DNA replication of the two viral strains demonstrated that the amount of viral DNA was slightly less at $1 \mathrm{~h}$ pi than at $30 \mathrm{~min}$ pi in both viruses (Figure 6). We can only speculate about this phenomenon. It is possible that the cellular DNases digest some proportion of the infecting 
viral DNA molecules. DNA synthesis was initiated between 2 and $4 \mathrm{~h}$ pi in the $w t$ virus. There was a decrease in the rate of multiplication of the DNA within the interval 4-6 h pi, which might be due to the switch between the theta-type and rolling circle-type of replications. A high rate of amplification of DNA molecules was observed between 6 and $12 \mathrm{~h}$ pi, followed by a slow decrease in the copy number of the wt viral DNA. The decrease in the amount of viral DNA in the late stage of viral infection is explained by the egress of mature virions from the infected cells. The onset of the DNA replication of the mutant virus exhibited a significant delay relative to that of the $w t$ virus. The us1-KO DNA started replication $4 \mathrm{~h}$ later, and the rate of production of viral genomes was lower than that of the $w t$ virus within the period 6-12 $\mathrm{h}$ pi. The copy numbers of the viral genomes were almost exactly the same at their peaks in the two viruses, but the maximum amount was reached $6 \mathrm{~h}$ later in the mutant virus. The mechanism whereby ICP22 affects the DNA replication remains to be determined, but it may be associated with the effect of the us1 mutation on the delay of the onset of the general gene expression. Since the expressions of several key viral factors, such as the IE180 and the EP0, were profoundly changed in consequence of the mutation, the effect of ICP22 on the DNA replication, at least in part, must be indirect.

\section{Investigation of the expression kinetics of PRV genes in the mutant and wt viruses through the use of $\mathrm{R}$ values normalized to the relative copy numbers of the DNA}

We analyzed the viral gene expressions by normalizing the amounts of transcripts to the relative copy number of the DNA molecules, which allows a comparison of the transcriptional activity with the corresponding copy number of the DNA at different time points. The ratios of the normalized $R$ values $\left(R_{\text {rn }}=R_{n \text {-us1KO }} / R_{n-w t}\right)$ indicated that the level of transcripts in most genes was lower in the mutant than in the $w t$ virus within the period $0.5-1 \mathrm{~h}$ pi with two exceptions ( $u l 21$ and $u l 24$ at $30 \mathrm{~min} \mathrm{pi).} \mathrm{This} \mathrm{ten-}$ dency was substantially reversed at $4 \mathrm{~h}$ pi, when the genes of the mutant virus were more active than those of the $w t$ virus (Figure 7). Some early genes (ep0, ul9, ul30, and ul52) produced $>100$ times more transcripts from a single copy of the genes in the mutant than in the $w t$ virus. There was not such a large difference between the nonnormalized genes of the two viruses. The switch from low to high amounts of transcripts occurred much later in the mutant virus, the exact time of the switch varying from gene to gene (Figure 2). The average expression from a single gene was very low at $30 \mathrm{~min}$ and $1 \mathrm{~h}$ pi in the us1$K O$ virus as compared with the $w t$ virus, but it had become $>50$ times higher $\left(R_{\text {us1KO }} / R_{\mathrm{wt}}\right)$ by $4 \mathrm{~h}$ pi (Figure $8 \mathrm{~A}$ ). This value was especially high for the average $\mathrm{E}$ genes (101.5), lower for the E/L genes (53), and much lower for the L genes (15.2). This phenomenon obviously resulted from the delayed onset of DNA synthesis in the us1-KO virus, which suggests that DNA replication exerts a severe constraint on the transcription. Indeed, the gene transcription rate was profoundly decreased after the initiation of DNA synthesis (Figures $8 \mathrm{~A}$ and $8 \mathrm{~B}$ ). In the $w t$ virus, the average rate of gene transcription was 33 fold less at $4 \mathrm{~h}$ pi than at $2 \mathrm{~h}$ pi $\left(\mathrm{R}_{4 \mathrm{~h}} / \mathrm{R}_{2 \mathrm{~h}}\right)$, and remained low or even became lower. In the mutant virus, the decrease in the rate of transcription started later (from $4 \mathrm{~h} \mathrm{pi}$ ), and occurred gradually, not suddenly as in the $w t$ virus. The steepest drop in gene expression in the interval 4-6 h pi did not coincide with the highest rate of DNA synthesis, at 6 to $8 \mathrm{~h}$ pi, which suggests that the inhibition of overall gene expression is not directly controlled by DNA replication itself, as it was suggested by Huvet and colleagues [31]. From data obtained in the analysis of human cells, those authors proposed novel gene expression regulatory mechanism, based on the collision of the transcriptional and DNA replication machineries. However, those results could not be reproduced by Necsulea and colleagues [32]. Although, it is generally conceived that the switch in expression from the $\mathrm{E}$ to the L kinetic class of genes is triggered by DNA replication, Figure $8 \mathrm{~B}$ clearly shows that the maximum expression as regards the individual $\mathrm{L}$ genes occurs at $2 \mathrm{~h} \mathrm{pi}$, i.e. before the onset of DNA replication, which casts severe doubt on the validity of this idea.

\section{Normalization of the $\mathrm{R}$ values to the relative DNA copy number leads to the $i e 180$ gene correlating with the other viral genes in the wt background}

Normalization of the R values of the cDNAs to the copy number of the viral DNAs leads to a very strong correlation between the ie180 and the other genes in both genetic backgrounds (Additional file 5B). It is noteworthy that the strength of the correlation between the ie180 and the different kinetic classes of genes is the opposite that in the non-normalized case. These data suggest that the synchronism in the transcription between the IE180 transactivator and the other genes is indicative of a real correlation. However, since the DNA replication imposes a severe constraint on the transcription of each gene, including the ie180 gene, the high correlation between the normalized data could possibly be merely a statistical curiosity without any functional significance. Additional file 6 shows the $\mathrm{R}$ values of $i e 180$, the average total viral genes and the average of various kinetic classes of genes normalized to the DNA copy number at different time points of infection.

\section{Conclusions}

Our kinetic data show that the abrogation of the us1 function leads to a significant reduction of transcription in every kinetic class of genes in the first hour pi. In the 
period 1 to $6 \mathrm{~h}$ pi, the L genes are selectively downregulated, and the E genes are later up-regulated in the us1-KO background relative to the $w t$ virus. The questions as to whether the ICP22 protein exerts a direct effect upon the gene expression and, if so, at what levels remains to be answered. It is noteworthy that deletion of the vhs gene resulted in a similar overall expression pattern as that observed in the us1-KO virus, and in an expression profile complementary to those in the ep0-KO virus. Interestingly, epO was the gene that was most affected in both the us1 and vhs knockout viruses (highly elevated expressions), which might imply that EPO could be a common link in the determination of the overall gene expression profile. We observed a strong inhibition of transcription during DNA replication. The question arises of whether the process of DNA replication itself could exert this inhibitory effect. In the mutant virus, the main inhibition in gene expression occurs between 4 and $6 \mathrm{~h}$ pi, while DNA synthesis exhibits the highest rate $2 \mathrm{~h}$ later. This suggests that the decrease in gene expression is not, or not only, a result of the potential collision of the transcription and DNA replication machineries. We have earlier shown that disruption of the function of the vhs gene of the virus results in synchronization of the gene expression profile of the ie180 and the remaining PRV genes [30]. We obtained very similar results with the us 1 gene-deleted PRV: the expression of the ie180 gene correlated with those of the other PRV genes in the mutant virus, which was not the case for the $w t$ virus. The question may be posed of whether this correlation is directly associated with the disruption of the us1 gene function, or is rather caused by the delay of DNA replication.

\section{Methods}

\section{Cells and viruses}

Monolayer cultures of porcine kidney epithelial cells (PK-15) were used for the propagation of the pseudorabies virus. Cells were grown in DMEM (Sigma Aldrich), supplemented with $5 \%$ fetal bovine serum (Gibco) and $80 \mu \mathrm{g}$ of gentamicin per ml (Invitrogen) at $37^{\circ} \mathrm{C}$ in the presence of $5 \% \mathrm{CO}_{2}$. The Kaplan $w t$ strain of PRV was used as the parental strain for the generation of the us1-null mutant virus (us1-KO).

\section{Construction of the us1 gene knockout virus}

The Ka-us1-KO (us1-KO, in short) virus was generated as follows. As a first step the BamHI-10 fragment of PRV was isolated from the gel, and was then subcloned to pRL525 [33], resulting in the generation of pRL525B10. This plasmid was used as a template for the PCR amplification of the two arms of the flanking sequences providing homology with the target viral genomic region. A green-fluorescent protein (GFP) gene expression cassette (Clontech) was inserted into the unique Ecl136II site of the targeting sequence, resulting in pUS1-gfp, which was used as the transfer plasmid for the generation of the knockout virus. The linearized transfer plasmid was transfected along with the purified $w t$ viral DNA into PK-15 cells. The recombinant virus was generated by homologous recombination, then isolated and plaque-purified on the basis of the fluorescence. Rescued viruses were generated by using pUS1 as a transfer plasmid, which was co-transfected with the purified DNA of us1-KO to PK-15 cells. The revertant viruses were selected on the basis of the non-fluorescent plaque phenotype. Both mutant and rescued viruses were checked with DNA sequencing.

\section{Infection}

The virus stock used for the experiments was prepared by infecting PK-15 cells with low-dose viruses, followed by incubation of the cells until a complete cytopathic effect was observed. To assess the effect of the us1 gene deletion on the transcription kinetics of PRV, rapidlygrowing semi-confluent PK-15 cells were infected with the $w t$ or $u s 1-K O$ virus at a high multiplicity of infection [MOI; 10 plaque forming units (pfu)/cell], and incubated for $1 \mathrm{~h}$, which was followed by removal of the virus suspension and washing of the cells with PBS. Subsequently, fresh culture medium was added to the cells, which were further cultivated for an additional $0.5,1,2$, $4,6,8,12,18$ or $24 \mathrm{~h}$.

\section{Isolation of RNAs}

Infected or non-infected PK-15 cells were washed with PBS and harvested for RNA purification. Total RNA was isolated by using the NucleoSpin RNA II Kit (MachereyNagel GmbH and Co. KG) as recommended by the supplier. Briefly, harvested cells were collected by low-speed centrifugation, and lysed in the buffer included in the kit. Samples were treated with RNase-free rDNase solution (included in the Kit) to remove potential genomic DNA contamination. As the next step, the potential residual DNA contamination was removed by using Turbo DNase (Ambion Inc.). Subsequently, RNA samples were eluted in RNase-free water (supplied with the kit), resulting in a total volume of $60 \mu \mathrm{l}$ of RNA solution. RNA concentrations were measured spectrophotometrically in a BioPhotometer Plus (Eppendorf). The RNA solution was stored at $-80^{\circ} \mathrm{C}$ until use.

\section{Reverse transcription}

Total RNA samples were reverse-transcribed by using gene-specific primers, and SuperScript III reverse transcriptase (Invitrogen) as described in our earlier reports $[9,34]$. Briefly, RT mixtures containing total RNA, primer, SuperScript III enzyme, buffer, dNTP mix and 
RNase inhibitor (RNAsin, Promega) were incubated at $55^{\circ} \mathrm{C}$ for $1 \mathrm{~h}$. The amplification of the first-strand cDNA synthesis was terminated by keeping the samples at $70^{\circ} \mathrm{C}$ for $15 \mathrm{~min}$. The cDNAs were diluted 10-fold with nuclease-free water (Promega Corp.) and the solutions were stored at $-80^{\circ} \mathrm{C}$ until use.

\section{Real-time PCR}

SYBR Green-based (Absolute QPCR SYBR Green Mix, Thermo Scientific) quantitative real-time PCRs were performed on the first-strand cDNAs in a real-time PCR cycler (Rotor-Gene 6000, Corbett Life Sciences), as described in our previous studies [9,30]. The specificity of the reverse transcription and the PCR reactions was ensured by using no-RT, no-primer, and no-template controls. The accuracy of sampling was guaranteed by using $28 \mathrm{~S}$ rRNA of swine as loading control. The specificity of the PCR products was confirmed by melting point analysis, PAGE and/or DNA sequencing.

\section{DNA sequencing}

We used pyrosequencing with a Pyromark Q24 pyrosequencer (Qiagen) to validate the mutation of the us1 gene and the specificity of the PCR products obtained in the kinetic experiments in the event of doubt.

\section{Data analysis}

The relative expression ratios ( $\mathrm{R}$ values) of the cDNAs of the PRV genes were calculated via the following formula:

$$
R=\frac{\left(E_{\text {sample6h }}\right)^{C t_{\text {sample } 6 h}}}{\left(E_{\text {sample }}\right)^{C t_{\text {sample }}}}: \frac{\overline{\left(E_{\text {ref } 6 h}\right)^{C t_{\text {ref } 6 h}}}}{\left(E_{\text {ref }}\right)^{C t_{\text {ref }}}}
$$

The cDNAs were all normalized to the cDNAs of the $28 \mathrm{~S}$ rRNAs of swine [9] by using the Comparative Quantitation module of the Rotor-Gene 6000 software (Version 1.7.28, Corbett Research), which automatically sets the thresholds and calculates the efficiency of PCR reactions. We used the average 6-h ECt values of the "samples", with those of the "references" as controls, as in our earlier works [9,30,34]. The $\mathrm{R}$ values of the viral DNAs were calculated similarly; the 6-h ECt values were taken as the control, and porcine $28 \mathrm{~S}$ rRNA gene was used as the reference. The effect of deletion of the us1 gene on the global gene expression was calculated by using $R_{r}$, the ratio of the $R$ values of the us1 mutant and the $w t$ viruses $\left(\mathrm{R}_{\mathrm{r}}=\mathrm{R}_{\mathrm{us} 1 \mathrm{KO}} / \mathrm{R}_{\mathrm{wt}}\right)$, where $R_{\mathrm{us} 1 K O}$ and $R_{\mathrm{wt}}$ are the $R$ values of a particular gene at a given time point in the us1-KO and $w t$ genetic background, respectively. All data were analyzed by using the average and the standard deviance functions of Microsoft Excel. Pearson's correlation coefficient was calculated for the analysis of the correlation between the expression kinetics of the genes, using the following formula:

$$
r=\frac{\sum_{i=1}^{n}\left(X_{i}-\bar{X}\right)\left(Y_{i}-\bar{Y}\right)}{(n-1) S_{x} S_{y}} \quad \text { [35]. The normalized } \mathrm{R}
$$

values were calculated by dividing the appropriate $R$ value of the cDNA by the $R$ value of the viral DNA measured at the same time for the same sample. The Pearson correlation coefficient is a number between -1 and +1 that measures the linear relationship between two variables, denoted here as $\mathrm{X}$ and $\mathrm{Y}$, which are the $\mathrm{R}$ values of two different genes or the average $R$ values of genes belonging in the same kinetic class in the same time interval. $\mathrm{X}$ and $\mathrm{Y}$ are the average values, $\mathrm{n}$ is the sample number, and SX and SY are the standard errors of the mean values for $\mathrm{X}$ and $\mathrm{Y}$, respectively. A positive value for the correlation indicates a positive association, while a negative value indicates an inverse association.

\section{Additional files}

Additional file 1: Comparison of the rates of increase of viral DNA during the first twelve hours of PRV infection. We observed similar dynamics in the growth rates of the DNA of the wild-type and rescued PRVs, while both differed significantly from those of the us1-mutant virus.

Additional file 2: Comparison of the transcription kinetics of the ie180 gene in the wild-type, us1-KO and us1-rescued PRV. This revealed that the kinetics of the rescued virus resembled that of the wildtype PRV, but differed significantly from that of the mutant virus.

Additional file 3: Comparison of the transcription kinetics of the ep0 gene in the wild-type, us1-KO and us1-rescued PRV. This revealed that the kinetics of the rescued virus resembled that of the wildtype PRV, but differed significantly from that of the mutant virus.

Additional file 4: The average relative expression ratios $(\bar{R})$. This table shows the $\bar{R}$ values for the total PRV genes (A) and for each kinetic class of viral genes (B) at different time points of infection.

Additional file 5: Correlations between the transcription of the ie180 gene and other PRV genes in wt and us1-KO backgrounds A. The viral genes are expressed in synchronism with the ie180 gene in the mutant virus, whereas the expressions are not correlated in the wt virus. B. Correlation between the transcription of the ie180 gene and other PRV genes in the $w t$ and us 1-KO backgrounds with the use of normalized $\mathrm{R}$ values. The expression of viral genes becomes correlated with the expression of iel 80 genes in the wt virus, too.

Additional file 6: The normalized average relative expression ratios $\left(\bar{R}_{n}\right)$ values This table shows the $\bar{R}_{n}$ values for the total PRV genes (A) and for each kinetic class of viral genes (B) at different time points of infection.

\section{Competing interests}

The authors declare that they have no competing interests.

\section{Authors' contributions}

IFT carried out the construction of the targeting plasmids, RNA purification, the reverse transcription reactions, the standard and real-time PCR and. DT carried out the DNA sequencing, and participated in the evaluation of the primary data. BB took part by performing the RT reactions and the real-time PCR. IP purified PRV RNA, propagated PK-15 cells and participated in the genotyping of the recombinant virus. NP carried out the agarose and polyacrylamide gel electrophoresis and participated in the propagation of cultured cells. ZB coordinated the study, propagated viruses, isolated the 
viral DNAs and isolated the recombinant viruses. All authors have read and approved the final manuscript.

\section{Acknowledgements}

We would like to thank Katalin Révész, Csilla Papdi and Margit Kisapáti for the technical assistance. This study was supported by grant TÁMOP-4.2.1.B09/1//KONV, TÁMOP-4.2.2/B-10/1-2010-0012, and Swiss-Hungarian

Cooperation Programme grant $\mathrm{SH} / 7 / 2 / 8$ to ZB.

Received: 23 June 2012 Accepted: 24 January 2013

Published: 29 January 2013

\section{References}

1. Aujeszky A: A contagious disease, not readily distinguishable from rabies, with unknown origin. Veterinarius 1902, 12:387-396.

2. Pomeranz L, Reynolds A, Hengartner C: Molecular biololgy of pseudorabies virus: Impact on neurovirology and veterinary medicine. Microbiology and Molecular Biology Reviews 2005, 69(3):462.

3. Boldogkoi Z, Balint K, Awatramani GB, Balya D, Busskamp V, Viney TJ, Lagali PS, Duebel J, Pasti E, Tombacz D, Toth JS, Takacs IF, Scherf BG, Roska B: Genetically timed, activity-sensor and rainbow transsynaptic viral tools. Nature Methods 2009, 6(2):127-130.

4. Enquist LW: Five Questions about Viral Trafficking in Neurons. Plos Pathogens 2012, 8(2):e1002472.

5. Boldogkoi Z, Nogradi A: Gene and cancer therapy-pseudorabies virus: a novel research and therapeutic tool? Curr Gene Ther 2003, 3(2):155-182

6. Prorok J, Kovacs PP, Kristof AA, Nagy N, Tombacz D, Toth JS, Ordog B, Jost N, Virag L, Papp JG, Varro A, Toth A, Boldogkoi Z: Herpesvirus-mediated delivery of a genetically encoded fluorescent $\mathrm{Ca}(2+)$ sensor to canine cardiomyocytes. J Biomed Biotechnol 2009, 2009:361795.

7. Boldogkoi Z, Bratincsak A, Fodor I: Evaluation of pseudorabies virus as a gene transfer vector and an oncolytic agent for human tumor cells. Anticancer Res 2002, 22(4):2153-2159.

8. Spivack J, Fraser N: Detection of Herpes-Simplex Virus Type-1 Transcripts during Latent Infection in Mice. J Virol 1987, 61(12):3841-3847.

9. Tombacz D, Toth JS, Petrovszki P, Boldogkoi Z: Whole-genome analysis of pseudorabies virus gene expression by real-time quantitative RT-PCR assay. BMC Genomics 2009, 10:491.

10. Boldogkoi Z, Braun A, Fodor I: Replication and virulence of early protein 0 and long latency transcript deficient mutants of the Aujeszky's disease (pseudorabies) virus. Microb Infect 2000, 2(11):1321-1328.

11. Schwartz J, Brittle E, Reynolds A, Enquist L, Silverstein S: UL54-null pseudorabies virus is attenuated in mice but productively infects cells in culture. J Virol 2006, 80(2):769-784.

12. Fuchs W, Ehrlich C, Klupp B, Mettenleiter T: Characterization of the replication origin (Ori(S)) and adjoining parts of the inverted repeat sequences of the pseudorabies virus genome. J Gen Virol 2000, 81:1539-1543

13. Zhang G, Leader D: The Structure of the Pseudorabies Virus Genome at the End of the Inverted Repeat Sequences Proximal to the Junction with the Short Unique Region. J Gen Virol 1990, 71:2433-2441.

14. Bowman JJ, Orlando JS, Davido DJ, Kushnir AS, Schaffer PA: Transient Expression of Herpes Simplex Virus Type 1 ICP22 Represses Viral Promoter Activity and Complements the Replication of an ICP22 Null Virus. J Virol 2009, 83(17):8733-8743.

15. Poffenberger K, Raichlen P, Herman R: In-Vitro Characterization of a Herpes-Simplex Virus Type-1 Icp22 Deletion Mutant. Virus Genes 1993, 7(2):171-186.

16. Purves F, Ogle W, Roizman B: Processing of the Herpes-Simplex Virus Regulatory Protein Alpha-22 Mediated by the U(I)13 Protein-Kinase Determines the Accumulation of a Subset of Alpha-Messenger Rnas and Gamma-Messenger Rnas and Proteins in Infected-Cells. Proc Natl Acad Sci US A 1993, 90(14):6701-6705.

17. Koppel R, Vogt B, Schwyzer M: Immediate-early protein BICP22 of bovine herpesvirus 1 trans-represses viral promoters of different kinetic classes and is itself regulated by BICPO at transcriptional and posttranscriptional levels. Arch Virol 1997, 142(12):2447-2464.

18. Rice S, Long M, Lam V, Schaffer P, Spencer C: Herpes-Simplex Virus Immediate-Early Protein Icp22 is Required for Viral Modification of Host Rna-Polymerase-li and Establishment of the Normal Viral Transcription Program. J Virol 1995, 69(9):5550-5559.
19. Long $M$, Leong $V$, Schaffer $P$, Spencer $C$, Rice S: ICP22 and the UL13 protein kinase are both required for herpes simplex virus-induced modification of the large subunit of RNA polymerase II. J Virol 1999, 73(7):5593-5604.

20. Advani S, Weichselbaum R, Roizman B: Herpes simplex virus 1 activates cdc2 to recruit topoisomerase II alpha for post-DNA synthesis expression of late genes. Proc Natl Acad Sci U S A 2003, 100(8):4825-4830.

21. Rice S, Long M, Lam V, Spencer C: Rna-Polymerase-li is Aberrantly Phosphorylated and Localized to Viral Replication Compartments Following Herpes-Simplex Virus-Infection. J Virol 1994, 68(2):988-1001.

22. Fraser KA, Rice SA: Herpes simplex virus immediate-early protein ICP22 triggers loss of serine 2-phosphorylated RNA polymerase II. J Virol 2007, 81(10):5091-5101.

23. Bastian TW, Livingston CM, Weller SK, Rice SA: Herpes Simplex Virus Type 1 Immediate-Early Protein ICP22 Is Required for VICE Domain Formation during Productive Viral Infection. J Virol 2010, 84(5):2384-2394.

24. Boldogkoi Z, Braun A, Medveczky I, Glavits R, Gyuro B, Fodor I: Analysis of the equalization of inverted repeats and neurovirulence using a pseudorabies virus mutant strain altered at the UI/Ir junction. Virus Genes 1998, 17(1):89-98.

25. Boldogkoi Z: Transcriptional interference networks coordinate the expression of functionally-related genes clustered in the same genomic loci. Frontiers in Genetics 2012, 3:00122.

26. Soong $\mathrm{R}$, Tabiti $\mathrm{K}$ : Detection of colorectal micrometastasis by quantitative RT-PCR of cytokeratin 20 mRNA. Proc Am Assoc Cancer Res 2000, 41:391. XP002149389.

27. Chambers J, Angulo A, Amaratunga D, Guo H, Jiang Y, Wan J, Bittner A, Frueh $\mathrm{K}$, Jackson M, Peterson P, Erlander M, Ghazal P: DNA microarrays of the complex human cytomegalovirus genome: Profiling kinetic class with drug sensitivity of viral gene expression. J Virol 1999, 73(7):5757-5766.

28. Tombacz D, Toth JS, Boldogkoi Z: Effects of deletion of the early protein 0 gene of pseudorabies virus on the overall viral gene expression. Gene 2012, 493(2):235-242.

29. Kramer T, Greco TM, Enquist LW, Cristea IM: Proteomic Characterization of Pseudorabies Virus Extracellular Virions. J Virol 2011, 85(13):6427-6441.

30. Tombacz D, Toth JS, Boldogkoi Z: Deletion of the virion host shut-off gene of pseudorabies virus results in selective upregulation of the expression of early viral genes in the late stage of infection. Genomics 2011, 98(1):15-25.

31. Huvet M, Nicolay S, Touchon M, Audit B, D'Aubenton-Carafa Y, Arneodo A, Thermes $C$ : Human gene organization driven by the coordination of replication and transcription. Genome Res 2007, 17(9):1278-1285.

32. Necsulea A, Guillet C, Cadoret J, Prioleau M, Duret L: The Relationship between DNA Replication and Human Genome Organization. Mol Biol Evol 2009, 26(4):729-741.

33. Elhai J, Wolk C: A Versatile Class of Positive-Selection Vectors Based on the Nonviability of Palindrome-Containing Plasmids that Allows Cloning into Long Polylinkers. Gene 1988, 68(1):119-138.

34. Toth JS, Tombacz D, Takacs IF, Boldogkoi Z: The effects of viral load on pseudorabies virus gene expression. Bmc Microbiology 2010, 10:311.

35. Campbell AM, Heyer L: Basic research with DNA microarray. In Discovering Genomics Proteomics and Bioinformatics. 2nd edition. Edited by Anonymous. San Francisco: CSHL Press; 2007:238-241.

doi:10.1186/1471-2199-14-2

Cite this article as: Takács et al:: The ICP22 protein selectively modifies the transcription of different kinetic classes of pseudorabies virus genes. BMC Molecular Biology 2013 14:2. 